\title{
Hydrogen Sulfide Attenuates Myocardial Hypoxia-Reoxygenation Injury by Inhibiting Autophagy via mTOR Activation
}

\author{
Jian Xiao ${ }^{\mathrm{a}}$ Xiaoyan Zhu ${ }^{\mathrm{b}}$ Bo Kang ${ }^{\mathrm{a}}$ Jibin $\mathrm{Xu}^{\mathrm{a}}$ Lihui Wu $\mathrm{W}^{\mathrm{a}}$ Jiang Hong ${ }^{\mathrm{a}}$ \\ Yufeng Zhang ${ }^{a}$ Xin $\mathrm{Ni}^{\mathrm{b}}$ Zhinong Wang ${ }^{\mathrm{a}}$ \\ aDepartmentof Cardiothoracic Surgery, Changzheng Hospital, Second Military Medical University, \\ Shanghai, 'bepartment of Physiology, Second Military Medical University, Shanghai, China
}

\section{Key Words}

Autophagy $\cdot \mathrm{mTOR} \cdot \mathrm{H}_{2} \mathrm{~S} \cdot$ Hypoxia-reoxygenation $\cdot$ Cardiomyocyte

\begin{abstract}
Background: Autophagy plays a significant role in myocardial ischemia reperfusion (IR) injury. Hydrogen sulfide $\left(\mathrm{H}_{2} \mathrm{~S}\right)$ has been demonstrated to protect cardiomyocytes against IR injury, while whether it has anti-autophagy effect has not been known. The aim of this study was to investigate whether $\mathrm{H}_{2} \mathrm{~S}$ regulates autophagy during IR injury and its possible mechanism. Methods and Results: The cardiomyocytes of neonatal rats were randomized into Con, hypoxia-reoxygenation ( $\mathrm{HR}$ ) and $\mathrm{H}_{2} \mathrm{~S}$ protection groups. The severity of cell injury was evaluated by cell vitality (MTT) and lactate dehydrogenase (LDH) release assays, and autophagy level was evaluated by flow cytometry and the assessment of autophagy-related gene (Atg) expression, such as that of Beclin1 and LC3-II. In response to $\mathrm{H}_{2} \mathrm{~S}$,Beclin1 and LC3- I protein were found to be down-regulated andp-mTOR protein was found to be upregulated, together with an increase in cell vitalityand a decrease in LDH.Furthermore, to find out whether mTOR was involved in the protective effect of $\mathrm{H}_{2} \mathrm{~S}$, rapamycin, inhibiter of mTOR, was used with or without applying NaHS and HR. It was found that rapamycin attenuated the myocardiocyte protective effect of $\mathrm{H}_{2} \mathrm{~S}$. To demonstrate the effect of autophagy during HR injury, the cardiomyocytes were pre-treated with 3-MA, which is an autophagy inhibitor, cell injury was attenuated by 3-MA. Conclusions: $\mathrm{H}_{2} \mathrm{~S}$ plays a myocardial protective role against IR injury by regulating autophagy via mTOR activation.

Wang Zhinong, or Ni Xin

Department of Cardiothoracic Surgery, Changzheng Hospital, Second Military Medical University, Fengyang Road 415\#, Shanghai, (China); or Department of Physiology, Second Military Medical University, Xiangyin Road 800\#, Shanghai, (China)

E-Mail wangzn007@163.com or E-Mail nixphysiology@outlook.com
\end{abstract}




\section{Cellular Physiology Cell Physiol Biochem 2015;37:2444-2453

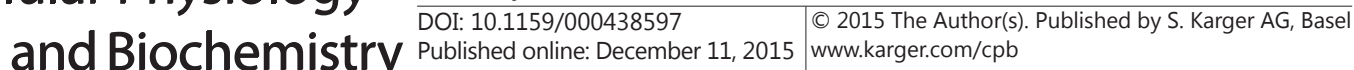 \\ Xiao et al.: $\mathrm{H}_{2} \mathrm{~S}$ Inhibits Myocardiocyte Autophagy via mTOR}

\section{Introduction}

Autophagy is a key mechanism for the maintenance ofcellular homeostasis, which has been associated with the degradation of damaged or unnecessary proteins and organelles [1]. Excessive autophagy may promote cell death, which is referred to as autophagic cell death or type II programmed cell death, together with apoptosis and necrosis [2]. There are 3 main autophagic pathways, namely macroautophagy, microautophagy, and chaperone-mediated autophagy. Unless otherwise specified, the term "autophagy" refers to macroautophagy [3]. Autophagy exists at low levels under physiological conditions but is up-regulated in response to stress, such as nutrient deprivation, hypoxia, ischemia-reperfusion (IR), mitochondrial dysfunction, and infection [4]. Autophagy provides protection in times of ischemia but can be detrimental during reperfusion [5]. Thus, we hypothesized that it could be beneficial for cardiomyocytes to attenuate autophagy induced by IR.

Hydrogen sulfide $\left(\mathrm{H}_{2} \mathrm{~S}\right)$ is recognized as an important signaling molecule in mammalian systems, as nitric oxide (NO) and carbon monoxide (CO) [6]. It is endogenously synthesized by cystathionine $\beta$-synthase (CBS), cystathionine $\gamma$-lyase (CSE), and 3 -mercaptopyruvate sulfurtransferase (3-MST) [7, 8]. In the heart, little CBS is present, whereas CSE is plentiful. It has been demonstrated that $\mathrm{H}_{2} \mathrm{~S}$ protects the myocardium against IR injury by inhibiting apoptosis via a mechanism that involves PI3K/Akt $[9,10]$. However, it is also known that autophagy may induce cell death when apoptosis is inhibited [3].

The present study was undertaken to determine whether $\mathrm{H}_{2} \mathrm{~S}$ protects the myocardium against IR injury by inhibiting autophagy in a dose-dependent manner. Our study shows that the inhibition of autophagy induced by $\mathrm{H}_{2} \mathrm{~S}$ is mainly dependent on mTOR activation.

\section{Material and Methods}

\section{Animal care}

All animal experiments were approved by the Animal Research Ethics Committee of the Second Military Medical University, Shanghai, China, andconformed to the Guide for the Care and Use of Laboratory Animals of the US National Institutes of Health.

\section{Cell culture and experimental protocols}

Neonatal cardiomyocytes were prepared from the heart of SD rats less than 3 days old [11]. On the 4th day, the cardiomyocytes were divided to three groups: the control group (Con), in which the cells were cultured in DMEM in 5\% $\mathrm{CO}_{2}$ and $95 \%$ air for $24 \mathrm{~h}$; the hypoxia-reoxygenation (HR) group, in which the cells were cultured in $1 \% \mathrm{O}_{2}, 5 \% \mathrm{CO}_{2}$ and $94 \% \mathrm{~N}_{2}$ atmosphere for $24 \mathrm{~h}$, and $5 \% \mathrm{CO}_{2}$ and $95 \%$ air for $6 \mathrm{~h}$; and the $\mathrm{H}_{2} \mathrm{~S}$ group, in which the cells were first treated with different concentrations of NaHS $(10 \mu \mathrm{M}, 30 \mu \mathrm{M}, 50 \mu \mathrm{M}$, $100 \mu \mathrm{M}) 30 \mathrm{~min}$ before HR and then followed the same protocol as the HR group. To determine whether mammalian target of rapamycin (mTOR) was involved in the protective effect of $\mathrm{H}_{2} \mathrm{~S}$, different doses of rapamycin (20 nM, $50 \mathrm{nM}, 100 \mathrm{nM})$, an inhibitor of mTOR, were added 30 min before NaHS. To evaluate whether autophagy promoted cardiomyocyte death, the cardiomyocytes was treated with different doses (1 mM, 2 mM, 5 mM) of 3-methyladenine (3-MA), an autophagy inhibitor [5], 30 min before HR. Cell vitaility and LDH releasewere measured after HR.

\section{MTT assay}

The MTT method was used to evaluate cardiomyocyte vitality. The absorbance of the purple solution was determined at $450 \mathrm{~nm}$ using a microtiter plate reader (Bio-Rad, USA).

Counting of autophagic vacuoles

Cardiomyocytes $\left(1 \times 10^{4}\right.$ cells per well) were incubated in $50 \mu \mathrm{M}$ monodansylcadaverine (MDC, Sigma) at $37^{\circ} \mathrm{C}$ for $1 \mathrm{~h}$. Cells were then washed 3 times with $\mathrm{PBS}$ at $37^{\circ} \mathrm{C}$ and fixed in $4 \%$ paraformaldehyde for $30 \mathrm{~min}$. After fixation, cells were washed 3 times with PBS. Autophagic vacuoles were analyzed by flow cytometryin the presence of Indo-1 (blue) dye with excitation laser set at $355 \mathrm{~nm}$ and detection of the fluorescence intensity at $488 \mathrm{~nm}$. 


\section{Cellular Physiology Cell Physiol Biochem 2015;37:2444-2453 \begin{tabular}{l|l|l} 
and Biochemistry $10.1159 / 000438597$ & (c) 2015 The Author(s). Published by S. Karger AG, Basel \\
Published online: December 11, 2015 & www.karger.com/cpb
\end{tabular} \\ Xiao et al.: $\mathrm{H}_{2} \mathrm{~S}$ Inhibits Myocardiocyte Autophagy via mTOR}

Quantitative real-time RT-PCR

The total RNA of cardiomyocytes was extracted using TRIzol reagent and reverse transcribed according to the manufacturer's instructions (Fermentas, CA). The annealing temperature of $\beta$-actin and Beclin 1 was set at $58^{\circ} \mathrm{C}$. The relative expression of both target (Beclin1, Atg5) and housekeeping ( $\beta$-actin) genes was determined using the threshold cycle (Ct) method based on the $2^{-\Delta \Delta \mathrm{Ct}}$ equation. The following sense and antisense primers were used: Beclin1 (accession number NM_001034117): 5'-GGCAGTGGCTCCTATT-3' and 5'-GGCGTGCTGTGCTCTGAAAA -3', Atg5 (accession number NM_001014250): 5'-AGTGGAGGCAACAGAACC-3' and 5' -GACACGAACTGGCACATT-3'.

\section{Westernblotting}

Protein concentration was determined using the BCA protein assay kit according the manufacturer's protocol. Equal amounts of protein $(50 \mu \mathrm{g})$ from the cardiomyocytes were subjected to Western blot analysis to evaluate the expression of LC3 (Sigma), mTOR, and p-mTOR(Ser-2448) (Cell Signaling Technology Inc, USA) using theECL detection kit (Amersham Biosciences, Piscataway, NJ). The autophagy results are presented as a ratio of LC3- II /LC3-I.

In vivo rat model and experimental protocols

SD rats (250-300 g) were anesthetized with $10 \%$ chloral hydrate $(300 \mathrm{mg} / \mathrm{kg}$, i.p.) before endotracheal intubation. IR was induced by ligating the left anterior descending artery (LAD) as previously reported [12]. Thirty rats were randomized to five groups: the control group, in which the rats underwent thoracotomy without ligation; the IR group, in which the rats were subjected to ischemia for $30 \mathrm{~min}$ and reperfusion for $3 \mathrm{~h}$; the $10 \mu \mathrm{M}$ NaHS group, in which the rats were treated with $10 \mu \mathrm{M} / \mathrm{kg}$ (body weight) NaHS by intraperitoneal injection $30 \mathrm{~min}$ before IR, and then followed the same protocol as the IR group; the $30 \mu \mathrm{M}$ NaHS group, in which the rats were treated with $30 \mu \mathrm{M} / \mathrm{kg}$ NaHS by intraperitoneal injection 30 min before IR, and then followed the same protocol as the IR group; the $100 \mu \mathrm{M}$ NaHS group, in which the rats were treated with $100 \mu \mathrm{M} / \mathrm{kg} \mathrm{NaHS}$ by intraperitoneal injection $30 \mathrm{~min}$ before IR, and then followed the same protocol as the IR group.

Statistical analysis

Quantitative data are presented as the mean \pm standard error (SEM). Statistical significance was determined by one-way ANOVA. Significance was established at the $\mathrm{P}<0.05$ level.

\section{Results}

Cardiomyocytes were injured by $\mathrm{HR}$, but $\mathrm{H}_{2} \mathrm{~S}$ attenuated cell injury in vitro

Cardiomyocyte viability was decreased and LDH release was increased after HR. To evaluate the protective effect of $\mathrm{H}_{2} \mathrm{~S}$, cardiomyocytes were treated with $\mathrm{H}_{2} \mathrm{~S}$ before HR. Compared with the HR group, cell vitality was increased and LDH release was decreased after HR. These effects were concentration dependent (Fig. 1).

Cardiomyocyte autophagy induced by $\mathrm{HR}$ was attenuated by $\mathrm{H}_{2} \mathrm{~S}$ in vitro

To assess the autophagy ratio of myocardiocytes, the amount of autophagosome was measured by flow cytometry. It was found that autophagy induced by HR was attenuated by $\mathrm{H}_{2} \mathrm{~S}$ (Fig. 2). Furthermore, to evaluate cardiomyocyte autophagy, Beclin1 mRNA levels were determined by RT-PCR and the levels of Beclin1 and LC3 proteins, autophagosome markers, was determined by westernblotting. The ratio of LC3- II / I was used to measure the relative autophagy level of cardiomyocytes. Beclin 1 mRNA, Beclin 1 protein and the ratio of LC3- II / I werefound to be up-regulated by HR but down-regulated by $\mathrm{H}_{2} \mathrm{~S}$. (Fig. 3).

\section{$\mathrm{H}_{2} \mathrm{~S}$ inhibited cardiomyocyte autophagy via mTOR activation}

To determine the mechanism of the anti-autophagic effect of $\mathrm{H}_{2} \mathrm{~S}$, we evaluated the protein expression of p-mTOR(Ser-2448). It was found that $\mathrm{H}_{2} \mathrm{~S}$ activated mTOR by up-regulatingp-mTOR protein phosphorylation, but the level of mTOR protein was not dysregulated. To examine the effect of mTOR on cardiomyocytes, the cells werepre-treated 


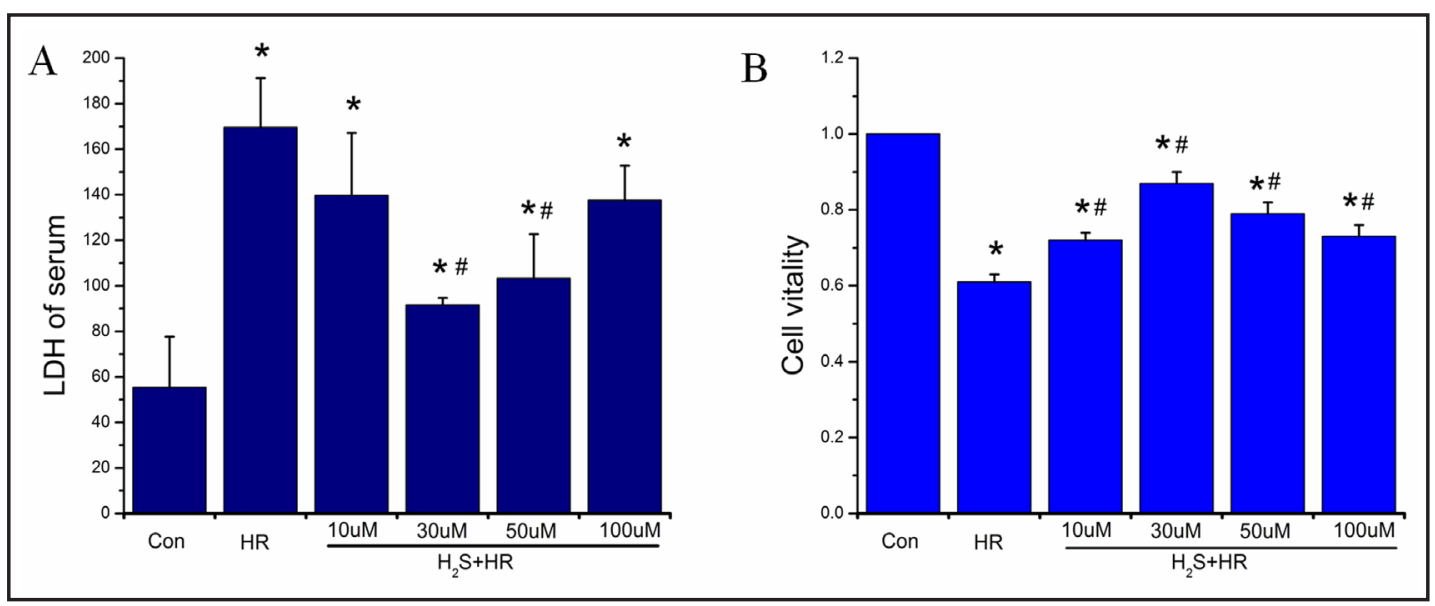

Fig. 1. Results of LDH in the cell culture serum and cardiomyocytes vitality measured with MTT method $(n=4)$. It was found that LDH was increased and cell vitality was decreased after HR. while compared with HR group, the injury induced by $\mathrm{HR}$ was attenuated by $\mathrm{H}_{2} \mathrm{~S}(\mathrm{n}=4)$. (A) LDH level of culture serum (from left to right and the follows were the same. ${ }^{*} \mathrm{P}=0.000,0.043,0.011,0.000$ vs Con, \# $\mathrm{P}=0.000,0.001$ vs HR); (B) Cardiomyocytes vitality measured with MTT method $\left({ }^{*} \mathrm{P}=0.000,0.000,0.002,0.000,0.000\right.$ vs Con, \# $\mathrm{P}=0.009$, $0.000,0.000,0.004$ vs HR).

Fig. 2. Results of cardiomyocyte autophagy ratio measured with flow cytometry system $(n=5)$. It was found that cardiomyocyte autophagy ratio was increased after HR, and was decreased by $\mathrm{H}_{2} \mathrm{~S}$. It was concentration dependent. $\quad\left({ }^{*} \mathrm{P}=0.000\right.$, $0.000,0.017,0.000$ vs Con, \# $\mathrm{P}=0.006,0.000,0.000$ vs HR).

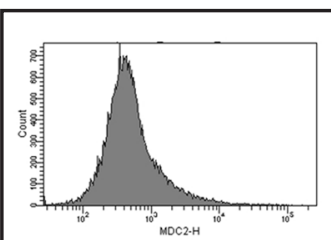

Con

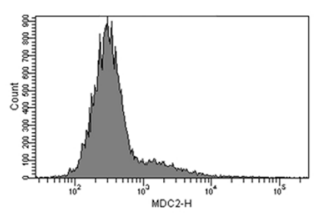

$30 \mathrm{uM} \mathrm{H}_{2} \mathrm{~S}+\mathrm{HR}$

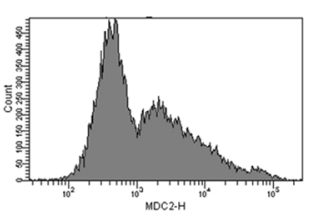

HR

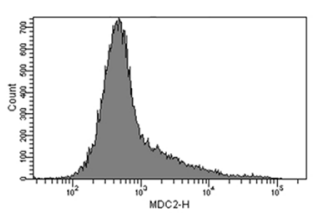

$50 \mathrm{uM} \mathrm{H}_{2} \mathrm{~S}+\mathrm{HR}$

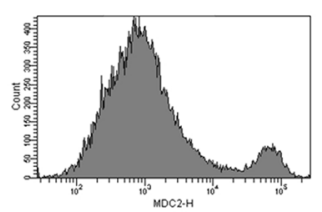

10uM H $\mathrm{H}_{2} \mathrm{~S}+\mathrm{HR}$

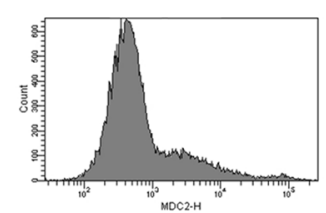

100uM H $\mathrm{H}_{2} \mathrm{~S}+\mathrm{HR}$

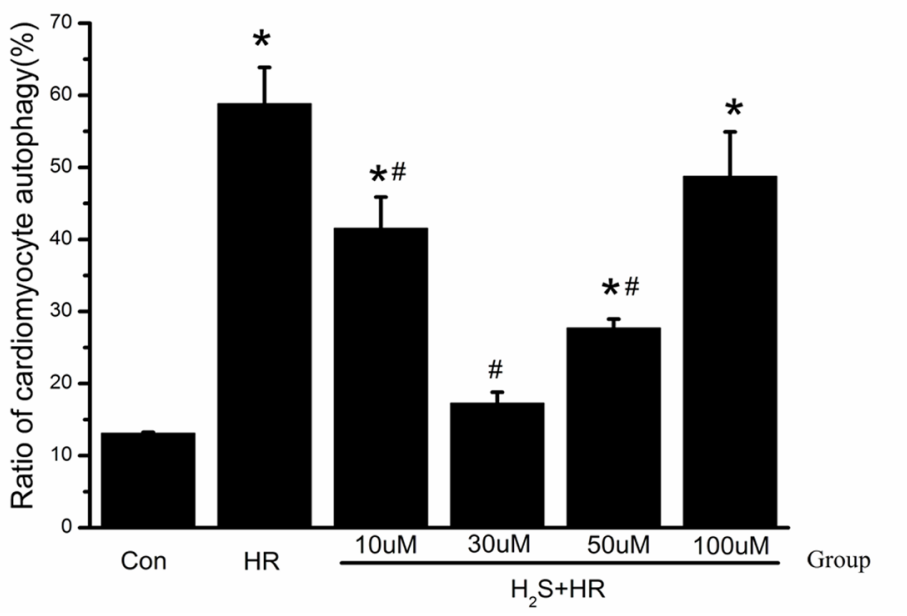

with rapamycin, a known inhibitor of mTOR,with or without HR. The ratio of LC3- II / I was increased by rapamycin, together with an increase inLDH release and a decrease in cell 


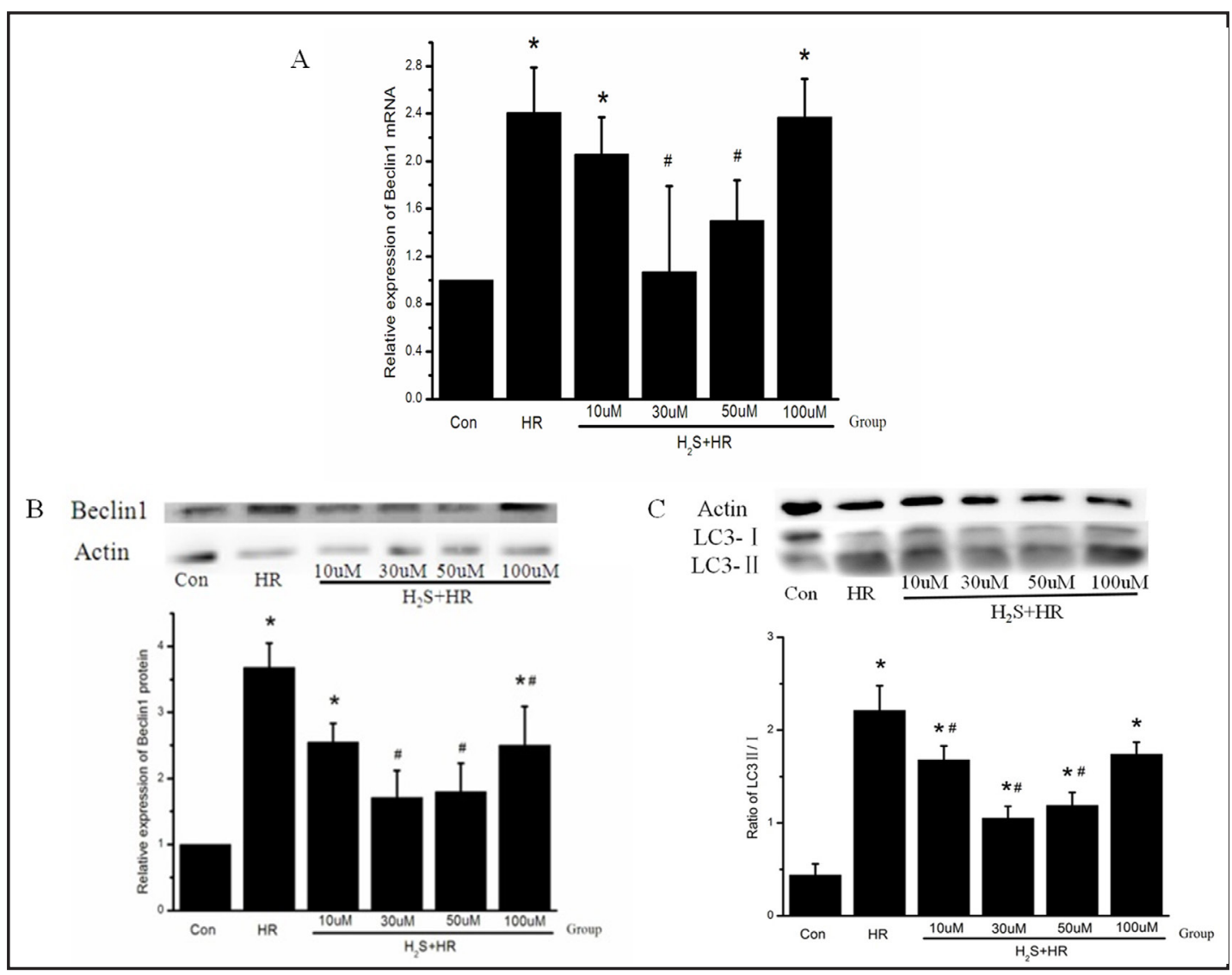

Fig. 3. Results of Atg mRNA and protein expression of cardiomyocyte $(n=5)$. It was found that Beclin 1 mRNA and protein were up-regulated after HR, together with LC3- II / I ratio increased. While NaHS pre-treatment could down-regulated Beclin1 expression and LC3- II / I ratio. (A) Result of Beclin1 mRNA expression $(* \mathrm{P}=0.002,0.013,0.002$ vs Con, $\# \mathrm{P}=0.002,0.031$ vs HR). (B) Result of Beclin 1 protein expression ( ${ }^{*} \mathrm{P}=0.000,0.012,0.014$ vs Con, \# $\mathrm{P}=0.002,0.013,0.047$ vs HR). (C) Results of LC3- I and LC3- II protein expression, and the ratio of LC3 - II / I was calculated to demonstrate the autophagy level. $(* \mathrm{P}=0.000,0.000$, $0.016,0.004,0.000$ vs Con, \# P=0.033, 0.000, 0.000 vs HR).

viability. Furthermore, to demonstrate whether $\mathrm{H}_{2} \mathrm{~S}$ inhibited cardiomyocyte autophagy via mTOR, the cardiomyocytes were treated with rapamycin 30 min before NaHS pre-treatment and HR. In that experiment, LDH was increased, and cell viability was decreased, compared with the $\mathrm{H}_{2} \mathrm{~S}$ group. In addition, the ratio of LC3- II / I was increased by rapamycin, which attenuated the inhibitory effect of $\mathrm{H}_{2} \mathrm{~S}$ on autophagy (Fig. 4).

Cardiomyocyte injury was attenuated by inhibiting autophagy with 3-MA

To determine whether autophagy was one type of cardiomyocyte death induced by HR, the cardiomyocytes were treated with 3-MA 30 min before HR. Cell vitality and LDH release were measured after HR. It was found that cell vitality was increased and LDH release was decreased by 3-MA in comparison tothe HR group (Fig. 5).

\section{LC3-II protein, Atg5 and Beclin1 mRNA were down-regulated by H2S in vivo}

It was found that Atg 5 and Beclin1 mRNA, which were up-regulated by IR, were downregulated by $\mathrm{H}_{2} \mathrm{~S}$ pre-treatment. The ratio ofLC3- II / LC3- I , which was increased in IR group, was also decreased in the $\mathrm{H}_{2} \mathrm{~S}$ pre-treatment groups; the ratio was optimal in the 30 $\mu \mathrm{M} / \mathrm{kg}$ group (Fig. 6).

\section{KARGER}




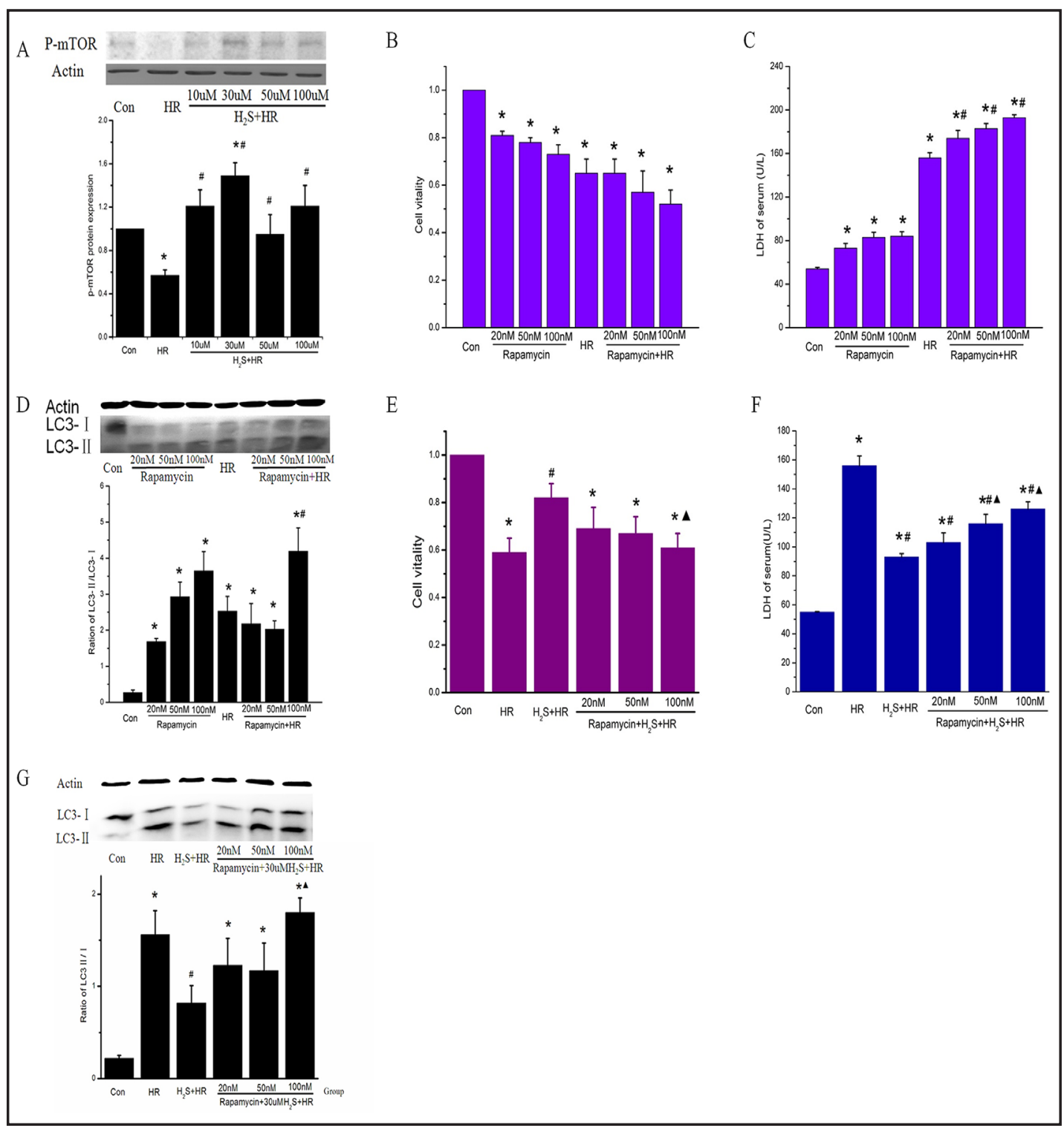

Fig. 4. Results of mTOR promoted autophagy and cardiomyocyte injury. (A) p-mTOR (ser2481) protein was up-regulated by $\mathrm{H}_{2} \mathrm{~S}$, while mTOR protein was unchanged. Comparing with Con group, p-mTOR was down-regulated in HR group. But it was up-regulated by NaHS pre-treatment before HR $\left({ }^{*} \mathrm{P}=0.020,0.009\right.$ vs Con, \# P=0.001, 0.000, 0.003, 0.001 vs HR). (B) Rapamycin promoted myocardiocytes injury. It was found that cell vitality was decreased after rapamycin treatment with or without $\mathrm{HR}\left({ }^{*} \mathrm{P}=0.018,0.006,0.001\right.$, $0.000,0.000,0.000,0.000$ vs Con). But there was no difference between HR and rapamycin pre-treatment groups. (C) LDH of serum was increased after rapamycin treatment with or without HR $\left({ }^{*} \mathrm{P}=0.007,0.000\right.$, $0.000,0.000,0.000,0.000,0.000$ vs Con). And comparing with HR group, LDH was more increased after HR with rapamycin pre-treatment (\# $\mathrm{P}=0.009,0.000,0.000$ vs HR). (D) Myocardiocytes autophagy was induced by rapamycin. $\left({ }^{*} \mathrm{P}=0.048,0.002,0.000,0.002,0.014,0.001,0.000\right.$ vs Con, \# $\mathrm{P}=0.021$ vs HR) (E) To find whether $\mathrm{H}_{2} \mathrm{~S}$ 's myocardium protection was mediated by mTOR activation, the cell was pretreatment by rapamycin 30min before NaHS, followed by HR. it was found that cell vitality was decreased by rapamycin comparing with $\mathrm{H}_{2} \mathrm{~S}$ group. ( ${ }^{*} \mathrm{P}=0.000,0.003,0.001,0.000$ vs Con, \# $\mathrm{P}=0.016$ vs HR, $\boldsymbol{\Delta} \mathrm{P}=0.028$ vs $\mathrm{H}_{2} \mathrm{~S}+\mathrm{HR}$ ) (F) LDH was increased by rapamycin comparing with $\mathrm{H}_{2} \mathrm{~S}$ group. $(* \mathrm{P}=0.000,0.000,0.000,0.000,0.000$ vs Con, \# P=0.000, $0.000,0.000,0.000,0.000$ vs HR, $\boldsymbol{\Delta} \mathrm{P}=0.003,0.000$ vs $\mathrm{H}_{2} \mathrm{~S}+\mathrm{HR}$ ) (G) Anti-autophagy effect of $\mathrm{H}_{2} \mathrm{~S}$ was attenuated by rapamycin. Comparing with $\mathrm{H}_{2} \mathrm{~S}$ group, ratio of LC3- II / I was increased by rapamycin $\left(* \mathrm{P}=0.001,0.006,0.009,0.000\right.$ vs Con, $\# \mathrm{P}=0.032$ vs $\mathrm{HR}, \boldsymbol{\Delta} \mathrm{P}=0.006$ vs $\left.\mathrm{H}_{2} \mathrm{~S}+\mathrm{HR}\right)$.

\section{KARGER}




\section{A}

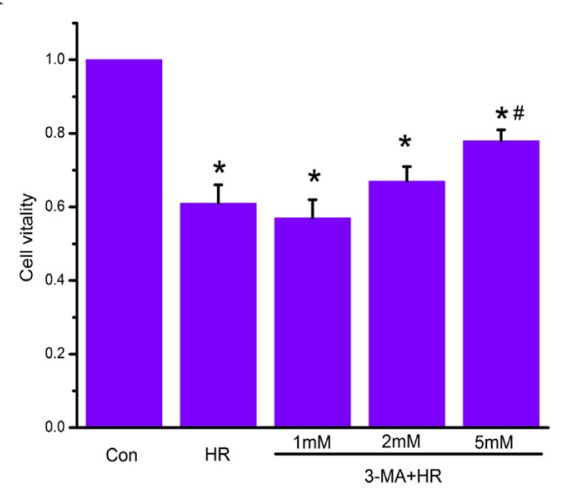

$\mathrm{B}$

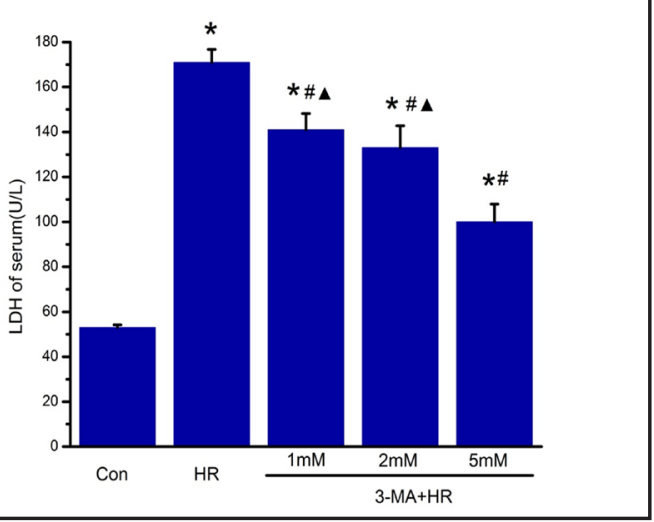

Fig. 5. Rresults of cardiomyocytes protection induced by 3-MA during HR. It was found that cell vitality was increased and LDH was decreased in 3-MA groups comparing with HR group. (A) Result of cardiomyocyte vitality measured with MTT ( ${ }^{*} \mathrm{P}=0.000,0.000,0.000,0.000$ vs Con, \# $\mathrm{P}=0.004$ vs HR). (B) Result of LDH of serum in different groups. $\left({ }^{*} \mathrm{P}=0.000,0.000,0.000,0.000\right.$ vs $\mathrm{Con}, \# \mathrm{P}=0.008,0.001,0.000$ vs HR, $\mathbf{\Delta} \mathrm{P}=0.001$, 0.002 vs $5 \mathrm{mM} 3-\mathrm{MA}+\mathrm{HR})$.

Fig. 6. Results of $L C 3$ II protein, Atg5 and Beclin1 mRNA expression of myocardium in vivo $(\mathrm{n}=6)$. It was found that Atg5 and Beclin1 mRNA and protein were up-regulated after IR, together with LC3- II / I ratio increased. While NaHS pre-treatment could down-regulated Atg5 and Beclin1 mRNA expression and LC3II / I ratio. (A) Result of Atg5 mRNA expression $\left({ }^{*} \mathrm{P}=0.009,0.03\right.$ vs Con, ( $\mathrm{P}=0.019$ vs IR). (B) Result of Beclin1 mRNA expression. $\quad\left({ }^{*} \mathrm{P}=0.027\right.$ vs Con, $\boldsymbol{\Delta} \mathrm{P}=0.03$ vs IR) (C) Results of LC3- I and LC3- II protein expression, and the ratio of LC3II / I was calculated to demonstrate the autophagy level. $\left({ }^{*} \mathrm{P}=0.002\right.$, 0.023 vs Con, \# $\mathrm{P}=0.005$, 0.015 vs IR).

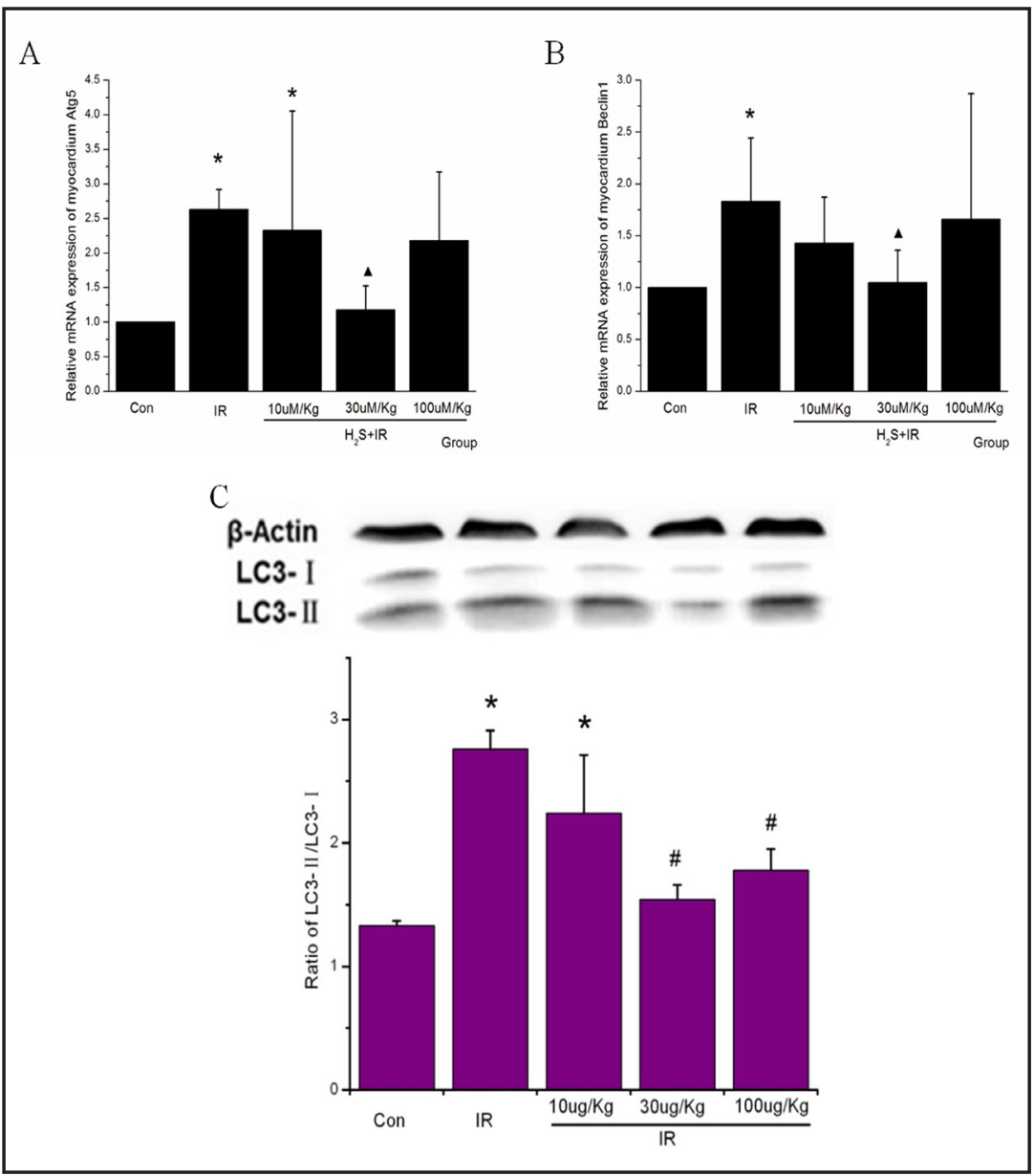

\section{Discussion}

Autophagy has been demonstrated to be involved in many physiological and pathological processes. When autophagy destroys the cytosol and organelles beyond a certain threshold, 


\section{Cellular Physiology Cell Physiol Biochem 2015;37:2444-2453

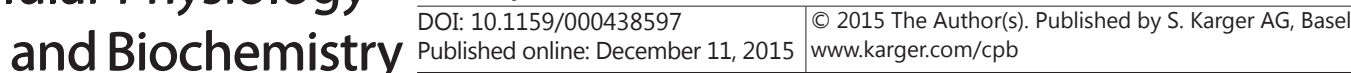 \\ Xiao et al.: $\mathrm{H}_{2} \mathrm{~S}$ Inhibits Myocardiocyte Autophagy via mTOR}

Fig. 7. The signal transduction mechanism of autophagy regulated by $\mathrm{H}_{2} \mathrm{~S}$.

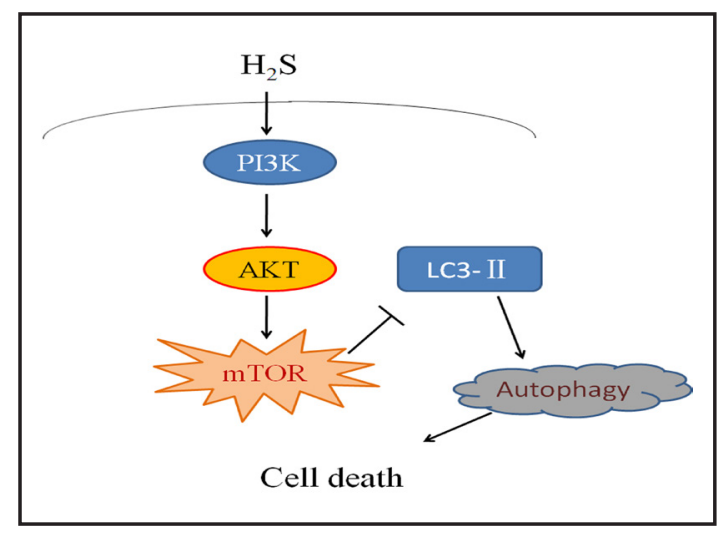

autophagic cell death will occur $[13,14]$. Excessive autophagy can be stimulated by nutrient starvation and growth factor deprivation when cells are unable to take up external nutrients [15]. Furthermore, autophagy can induce cell death when apoptosis is inhibited because the early stages of autophagy are required for apoptosis [3]. Under the condition of cardiac ischemia reperfusion injury (IR injury), the process of autophagy is activated in response to energy crisis and oxidative stress [1]. Although autophagy is protective during ischemia, it is detrimental during reperfusion followed by heart failure, as demonstrated by Matsui et al. [16].

The autophagy process is regulated by the autophagy-related genes (Atg), among which Beclin1 is needed for the autophagy vesicle nucleation step of autophagy [17]. In the mouse heart, the expression of Beclin 1 inthe area at risk was only slightly up-regulated by ischemia alone, but its expression was dramatically enhanced after IR. While autophagosomes were significantly attenuated in beclin $1^{+/}$mice, the size of myocardial infarction or the area at riskafter IR was also significantly smaller [16].

The formation of autophagosomesoccurs via two pathways, the Atg12-Atg5-Atg16 pathway and the Atg4-Atg7-Atg3 pathway. Conjugations led to the conversion of the soluble form of LC3 (LC3-I) to the autophagic vesicle-associated form (LC3-II), which is used as a marker of autophagy[18]. The ratio of LC3-II/ LC3-I was used to evaluate the autophagy level in most studies $[16,19]$. In our study, the autophagy level was determined by flow cytometry. It was found that the autophagy ratio was increased after HR, together with cell injury. Beclin1 and LC3-II, the autophagosome marker, were also up-regulated by HR. To demonstrate whether autophagy was detrimental during HR injury, 3-MA, which is the special inhibiter of autophagy, was used before HR. Cardiomyocyte vitality was found to be increased and LDH was found to be decreased by 3-MA. Therefore, the inhibition of autophagy may attenuate cardiac HR injury.

Recently, $\mathrm{H}_{2} \mathrm{~S}$, a gasotransmitter, has attracted wide attention because of its protective effect of brain, liver, kidney, heart, lung and other organs [20-24]. Many studies have demonstrated the cytoprotective effects of $\mathrm{H}_{2} \mathrm{~S}$ in myocardial IR injury [25, 26]. However, whether it could attenuate myocardiocyte autophagy has not been reported. We found that beclin 1 and LC3- II were down-regulated by $\mathrm{H}_{2} \mathrm{~S}$, together with a decrease inthe autophagy ratio after HR. Furthermore, the myocardiocyte vitality was increased and LDH release was decreased by $\mathrm{H}_{2} \mathrm{~S}$ during HR. These findings suggest that $\mathrm{H}_{2} \mathrm{~S}$ could attenuate not only apoptosis but also autophagy induced by IR. The same results were also found in heart injuries in rats inhaling tobacco smoke [7].The anti-autophagy mechanism of $\mathrm{H}_{2} \mathrm{~S}$ is still uncertain.

Mammalian target of rapamycin (mTOR), the down-stream effector of PI3K/Akt, plays a key role inautophagy regulation [27, 28]. mTOR negatively regulates autophagy [29], and autophagy would be activated by inhibiting mTOR activation[30, 31]. In the present study, the LC3-II protein, which was induced by HR, was down-regulated by $\mathrm{H}_{2} \mathrm{~S}$ in vitro. This was associated with an up-regulation of p-mTOR protein. To demonstrate whether $\mathrm{H}_{2} \mathrm{~S}$ inhibited cardiomyocyte autophagy induced by HR by activating the mTOR system, 


\section{Cellular Physiology Cell Physiol Biochem 2015;37:2444-2453

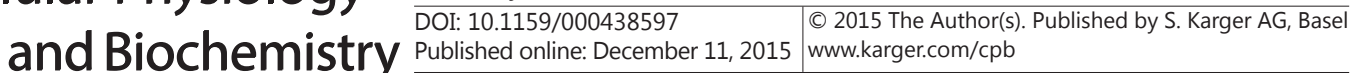 \\ Xiao et al.: $\mathrm{H}_{2} \mathrm{~S}$ Inhibits Myocardiocyte Autophagy via mTOR}

rapamycin, a specific mTOR inhibitor, was used with or without HR. The ratio of LC3-II/LC3I was found to be increased, together with a decrease incell vitality and an increase in LDH release. These results indicate that both autophagy and cell injury could be induced by the inhibition of mTOR. Furthermore, the treatment of cardiomyocytes with rapamycin $30 \mathrm{~min}$ before NaHS followed by HR attenuated the anti-autophagy and cell protective effects of $\mathrm{H}_{2} \mathrm{~S}$. These observations suggested that $\mathrm{H}_{2} \mathrm{~S}$ might protect cardiomyocytes against HR injury by inhibiting autophagy through the activation of the Akt/mTOR pathway.

\section{Conclusion}

In summary, our study demonstrated that $\mathrm{H}_{2} \mathrm{~S}$ played an important and protective role in cardiomyocytes by regulating autophagy against HR injury via the mTOR system (Fig. 7). Autophagy can spare cell death, or at least postpone it, because auto-digestion provides an alternative source of intracellular nutrients and clearance of destructive organelles in ischemia phase; but once autophagy over expressed, it might phagocytize more organelles and promote cell death. However, the exact function of autophagy in HR may be more complex.

\section{Acknowledgments}

This work was supported by Nature Science Foundation of Science and Technology Commission of Shanghai municipality (11JC1402402, 12ZR1454600 and 13430720901) and the National Nature Science Foundation of China (81170232, 81200181and 81270419).

\section{Disclosure Statement}

The authors declare no conflict of interest.

\section{References}

1 Ma S, Wang Y, Chen Y, Cao F: The role of the autophagy in myocardial ischemia/reperfusion injury. Biochim Biophys Acta 2015;1852:271-276.

2 Yuan H, Perry CN, Huang C, Iwai-Kanai E, Carreira RS, Glembotski CC, Gottlieb RA: Lps-induced autophagy is mediated by oxidative signaling in cardiomyocytes and is associated with cytoprotection. Am J Physiol Heart Circ Physiol 2009;296:H470-479.

3 Nishida K, Yamaguchi O, Otsu K: Crosstalk between autophagy and apoptosis in heart disease. Circ Res 2008;103:343-351.

4 Gustafsson AB, Gottlieb RA: Recycle or die: The role of autophagy in cardioprotection. J Mol Cell Cardiol 2008;44:654-661.

5 Gustafsson AB, Gottlieb RA: Autophagy in ischemic heart disease. Circ Res 2009;104:150-158.

6 Zhu XY, Liu SJ, Liu YJ, Wang S, Ni X: Glucocorticoids suppress cystathionine gamma-lyase expression and h2s production in lipopolysaccharide-treated macrophages. Cell Mol Life Sci 2010;67:1119-1132.

7 Zhou X, An G, Chen J: Hydrogen sulfide improves left ventricular function in smoking rats via regulation of apoptosis and autophagy. Apoptosis 2014;19:998-1005.

$8 \quad$ Xu SW, Liu ZP, Liu PQ: Targeting hydrogen sulfide as a promising therapeutic strategy for atherosclerosis. Int J Cardiol 2014;172:313-317.

9 Shi S, Li QS, Li H, Zhang L, Xu M, Cheng JL, Peng CH, Xu CQ, Tian Y: Anti-apoptotic action of hydrogen sulfide is associated with early jnk inhibition. Cell Biol Int 2009;33:1095-1101. 


\section{Cellular Physiology Cell Physiol Biochem 2015;37:2444-2453 \begin{tabular}{l|l|l|l|l}
\hline DOI: 10.1159/000438597 & $\begin{array}{l}\text { (c) 2015 The Author(s). Published by S. Karger AG, Basel } \\
\text { anww/karger.com/cpb }\end{array}$
\end{tabular} \\ Xiao et al.: $\mathrm{H}_{2} \mathrm{~S}$ Inhibits Myocardiocyte Autophagy via mTOR}

10 Yong QC, Lee SW, Foo CS, Neo KL, Chen X, Bian JS: Endogenous hydrogen sulphide mediates the cardioprotection induced by ischemic postconditioning. Am J Physiol Heart Circ Physiol 2008;295:H1330-H1340.

11 Jian X, Xiao-yan Z, Bin H, Yu-feng Z, Bo K, Zhi-nong W, Xin N: Mir-204 regulate cardiomyocyte autophagy induced by hypoxia-reoxygenation through lc3-ii. Int J Cardiol 2011;148:110-112.

12 He B, Xiao J, Ren AJ, Zhang YF, Zhang H, Chen M, Xie B, Gao XG, Wang YW: Role of mir-1 and mir-133a in myocardial ischemic postconditioning. J Biomed Sci 2011;18:22.

13 Lu L, Wu W, Yan J, Li X, Yu H, Yu X: Adriamycin-induced autophagic cardiomyocyte death plays a pathogenic role in a rat model of heart failure. Int J Cardiol 2009;134:82-90.

14 Rothermel BA, Hill JA: Autophagy in load-induced heart disease. Circ Res 2008;103:1363-1369.

15 Lum JJ, DeBerardinis RJ, Thompson CB: Autophagy in metazoans: Cell survival in the land of plenty. Nat Rev Mol Cell Biol 2005;6:439-448.

16 Matsui Y, Takagi H, Qu X, Abdellatif M, Sakoda H, Asano T, Levine B, Sadoshima J: Distinct roles of autophagy in the heart during ischemia and reperfusion: Roles of amp-activated protein kinase and beclin 1 in mediating autophagy. Circ Res 2007;100:914-922.

17 Yazdankhah M, Farioli-Vecchioli S, Tonchev AB, Stoykova A, Cecconi F: The autophagy regulators ambra1 and beclin 1 are required for adult neurogenesis in the brain subventricular zone. Cell Death Dis 2014;5:e1403.

18 Nishida K, Kyoi S, Yamaguchi O, Sadoshima J, Otsu K: The role of autophagy in the heart. Cell Death Differ 2009;16:31-38.

19 Jiao H, Zhang Z, Ma Q, Fu W, Liu Z: Mechanism underlying the inhibitory effect of apelin-13 on glucose deprivation-induced autophagy in rat cardiomyocytes. Exp Ther Med 2013;5:797-802.

20 Nicholson CK, Calvert JW: Hydrogen sulfide and ischemia-reperfusion injury. Pharmacol Res 2010;62:289297.

21 Yang CT, Zhao Y, Xian M, Li JH, Dong Q, Bai HB, Xu JD, Zhang MF: A novel controllable hydrogen sulfidereleasing molecule protects human skin keratinocytes against methylglyoxal-induced injury and dysfunction. Cell Physiol Biochem 2014;34:1304-1317.

22 Avanzato D, Merlino A, Porrera S, Wang R, Munaron L, Mancardi D: Role of calcium channels in the protective effect of hydrogen sulfide in rat cardiomyoblasts. Cell Physiol Biochem 2014;33:1205-1214.

23 Guo R, Wu K, Chen J, Mo L, Hua X, Zheng D, Chen P, Chen G, Xu W, Feng J: Exogenous hydrogen sulfide protects against doxorubicin-induced inflammation and cytotoxicity by inhibiting p38mapk/nfkappab pathway in h9c2 cardiac cells. Cell Physiol Biochem 2013;32:1668-1680.

24 Han Y, Zeng F, Tan G, Yang C, Tang H, Luo Y, Feng J, Xiong H, Guo Q: Hydrogen sulfide inhibits abnormal proliferation of lymphocytes via akt/gsk3beta signal pathway in systemic lupus erythematosus patients. Cell Physiol Biochem 2013;31:795-804.

25 Calvert JW, Jha S, Gundewar S, Elrod JW, Ramachandran A, Pattillo CB, Kevil CG, Lefer DJ: Hydrogen sulfide mediates cardioprotection through nrf2 signaling. Circ Res 2009;105:365-374.

26 Osipov RM, Robich MP, Feng J, Liu Y, Clements RT, Glazer HP, Sodha NR, Szabo C, Bianchi C, Sellke FW: Effect of hydrogen sulfide in a porcine model of myocardial ischemia-reperfusion: Comparison of different administration regimens and characterization of the cellular mechanisms of protection. J Cardiovasc Pharmacol 2009;54:287-297.

27 Mei Y, Thompson MD, Cohen RA, Tong X: Autophagy and oxidative stress in cardiovascular diseases. Biochim Biophys Acta 2015;1852:243-251.

28 Jung CH, Ro SH, Cao J, Otto NM, Kim DH: Mtor regulation of autophagy. FEBS Lett 2010;584:1287-1295.

29 Hiromitsu Takagi YM, Shinichi Hirotani, Sakoda H, Asano T, Sadoshima J: Ampk mediates autophagy during myocardial ischemia in vivo. Autophagy 2007;3:405-407.

30 Zhang XD, Wang Y, Zhang X, Han R, Wu JC, Liang ZQ, Gu ZL, Han F, Fukunaga K, Qin ZH: P53 mediates mitochondria dysfunction-triggered autophagy activation and cell death in rat striatum. Autophagy 2009;5:339-350.

31 Gurusamy N, Lekli I, Mukherjee S, Ray D, Ahsan MK, Gherghiceanu M, Popescu LM, Das DK: Cardioprotection by resveratrol: A novel mechanism via autophagy involving the mtorc2 pathway. Cardiovasc Res 2010;86:103-112. 